\section{Reduction in Central Line-Associated Bloodstream Infections in Patients with Burns}

Central line-associated bloodstream infections (CLABSIs) remain a threat to hospitalized patients. ${ }^{1}$ Patients with burn injuries are especially vulnerable to CLABSI, because the burn wound area can become colonized with pathogens, and prolonged hospital stays are common. In addition, large surface burns have a systemic immunomodulatory effect, such as skewing the immune system toward an interleukin 17mediated response. ${ }^{2}$ In this study, we evaluated CLABSI incidence trends in a large burn intensive care unit (ICU).

This study was conducted at the University of North Carolina (UNC) Hospitals using surveillance data from 1999 to 2012. UNC Hospitals is an 806-bed tertiary care academic facility. All patients admitted to the Jaycee Burn Center ICU, which is a 21-bed ICU dedicated to the care of severely ill patients with burns or extensive exfoliating skin conditions, were included. The number of ICU beds was 10 during the period 1999-2007 and increased to its current number of 21 beds in 2008. From 200 to 2009, a number of interventions directed toward decreasing the CLABSI rate were implemented (Table 1).

Infection control at UNC Hospitals is provided by 5 infection preventionists and 3 faculty members. Comprehensive hospital-wide surveillance was conducted using the most recent definitions recommended by the National Nosocomial Infection Surveillance ${ }^{3}$ and the National Healthcare Safety Network. ${ }^{4}$ Rates of CLABSI were calculated as the number of infections per 1,000 central line-days. Simple linear regression models (least-squares method) were used to examine decreases in the rate of CLABSI over time. Statistical signif- icance was assessed by comparing these regression lines with a line with a 0 slope.

The annual number of central line-days in the burn ICU increased from 1,493 days in 1999 to 3,223 days in 2012. At the same time the absolute number of CLABSI decreased from 21 CLABSI in 1999 to 7 CLABSI in 2012. This resulted in a substantial decrease in the rate of CLABSI in the burn ICU from 14.07 to 2.17 CLABSI per 1,000 central line-days (Figure 1). Over the period 2000-2012, we prevented an estimated 428 infections at a total cost savings of $\$ 9,947,576$, based on published cost data and 118 deaths. ${ }^{5}$ When evaluating the other ICUs during the study period, a reduction in the rate of CLABSI was also noted. Of note, since 2004, the rates of CLABSI in the burn ICU have been very similar to those in other ICUs. When the specific microbiologic etiologies were evaluated, a decrease in the number of CLABSI caused by Staphylococcus aureus was noted (Figure 1).

A sustained decreased CLABSI rate was observed in the burn ICU. Most interventions were implemented hospitalwide, and their effect was observed not only in the burn ICU, but in all ICUs, as previously reported. ${ }^{6}$ Some interventions were unique to the burn population. The frequency of line changes remains controversial in this population. The 2008 Society for Healthcare Epidemiology of America (SHEA)/ Infectious Diseases Society of America (IDSA) guidelines do not recommend routine line changes. ${ }^{7}$ A small study using historical controls was performed among burn patients to compare line changes every 3 days with line changes every 4 days. ${ }^{8}$ This study suggested that increasing the interval was associated with an increase in episodes of CLABSI. ${ }^{8}$ A survey of CLABSI-prevention practices across 51 adult burn units in the United States certified by the American Burn Association showed that $61 \%$ of burn units practice routine prophylactic line changes with an interval ranging from 3 to 7 days. ${ }^{9}$ In our cohort, line change practice included a guidewire

TAвLE 1. Interventions to Reduce Central Line-Associated Bloodstream Infections (CLABSIs) at University of North Carolina Hospitals, 2000-2009

\begin{tabular}{|c|c|}
\hline Year(s) & Intervention(s) \\
\hline 2000 & $\begin{array}{l}\text { Enhanced education of medical staff regarding central lines; addition of } 2 \% \text { chlorhexidine plus } 70 \% \text { isopropyl } \\
\text { alcohol for skin preparation to central line kits }\end{array}$ \\
\hline 2001 & Mandatory training for nurses on IV line site care and maintenance \\
\hline 2003 & $\begin{array}{l}\text { Central line changes over a guidewire every } 3 \text { days with use of a new site every } 6 \text { days becomes standard practice; } \\
\text { use of full body drape for line insertion and changes }\end{array}$ \\
\hline 2003-2005 & Introduction of antibiotic-impregnated central venous catheters for all patients \\
\hline 2004 & Enhanced nursing education on central line insertion and maintenance \\
\hline 2005 & Customized catheter-insertion kits \\
\hline 2006 & Universal glove and gown use for all patient encounters ${ }^{\mathrm{a}}$ \\
\hline 2007 & Implementation of the Institute for Healthcare Improvement bundle to prevent CLABSI \\
\hline 2009 & Use of chlorhexidine patch at insertion site \\
\hline
\end{tabular}

NOTE. IV, intravenous.

a Specific to the burn ICU. 


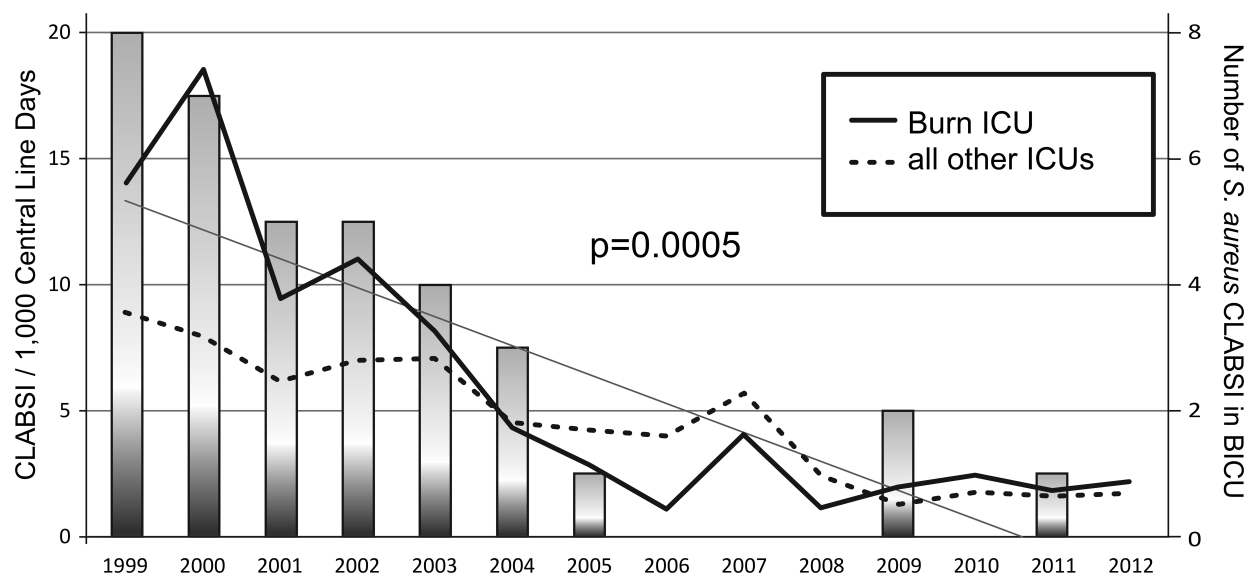

FIGURE 1. Trends in overall central line-associated bloodstream infection (CLABSI) rate and number of Staphylococcus aureus CLABSIs in the burn intensive care unit (BICU). The solid black line indicates the rate of CLABSI in the BICU, and the dashed line indicates the rate of CLABSI in all other intensive care units (ICUs). The gray line is the regression line of the rate of CLABSI in the BICU $(P=$ .0005). Shaded bars show the number of CLABSIs in the BICU per year caused by S. aureus.

exchange every 3 days and changing to a new site every 6 days.

Antibiotic-impregnated central venous catheters were used in our cohort as well as in $43 \%$ of burn units surveyed. ${ }^{9}$ The use of antibiotic-impregnated catheters is recommended in the 2008 SHEA/IDSA guidelines in hospital units with a CLABSI rate higher than the institutional goal, despite compliance with basic CLABSI-prevention practices. ${ }^{7}$

In 2006, universal glove and gown use was implemented in our burn ICU. This measure was not implemented in any other type of ICU in our hospital. The relative impact of this intervention on our CLABSI rate was likely modest, because most of the reduction in CLABSI rates occurred before this intervention. A recent cluster-randomized trial evaluated the impact of universal glove and gown use in non-burn ICUs. ${ }^{10}$ Their findings included a reduction in the acquisition of MRSA, but not of VRE. No changes in CLABSI rates were observed..$^{10}$ We noted that most of the reduction was driven by a dramatic reduction in gram-positive organisms, mirroring the national trends. ${ }^{1}$

In summary, we observed a large, sustained, and prolonged reduction in the rate of CLABSI in the burn ICU. This reduction was temporally associated with the implementation of a multifaceted proactive approach to CLABSI prevention.

\section{ACKNOWLEDGMENTS}

Potential conflicts of interest. All authors report no conflicts of interest relevant to this article. All authors submitted the ICMJE Form for Disclosure of Potential Conflicts of Interest, and the conflicts that the editors consider relevant to this article are disclosed here.

David van Duin, MD, $\mathrm{PhD} ;{ }^{1}$ Samuel W. Jones, $\mathrm{MD} ;{ }^{2,3}$ Lauren Dibiase, MS; ${ }^{4}$ Grace Schmits; ${ }^{3}$
Anne Lachiewicz, MD; ${ }^{1}$ Charles Scott Hultman, MD, MBA; ${ }^{5}$ William A. Rutala, PhD; ; ${ }^{1,4}$ David J. Weber, MD, MPH; $;^{1,4}$ Bruce A. Cairns, $\mathrm{MD}^{2,3}$

Affiliations: 1. Division of Infectious Diseases, University of North Carolina at Chapel Hill, Chapel Hill, North Carolina; 2. Department of Surgery, University of North Carolina at Chapel Hill, Chapel Hill, North Carolina; 3. North Carolina Jaycee Burn Center, Chapel Hill, North Carolina; 4. Department of Hospital Epidemiology, University of North Carolina Health Care, Chapel Hill, North Carolina; 5. Division of Plastic Surgery, University of North Carolina at Chapel Hill, Chapel Hill, North Carolina.

Address correspondence to David van Duin, MD, PhD, Division of Infectious Diseases, Department of Medicine, University of North Carolina, 130 Mason Farm Road, Mail Code CB\# 7030, Chapel Hill, NC 27599 (david_vanduin@med.unc.edu).

Received February 4, 2014; accepted March 29, 2014; electronically published June 20, 2014.

Infect Control Hosp Epidemiol 2014;35(8):1066-1068

(C) 2014 by The Society for Healthcare Epidemiology of America. All rights reserved. 0899-823X/2014/3508-0021\$15.00. DOI: 10.1086/677165

\section{REFERENCES}

1. Fagan RP, Edwards JR, Park BJ, Fridkin SK, Magill SS. Incidence trends in pathogen-specific central line-associated bloodstream infections in US intensive care units, 1990-2010. Infect Control Hosp Epidemiol 2013;34:893-899.

2. Neely CJ, Maile R, Wang MJ, Vadlamudi S, Meyer AA, Cairns BA. Th17 (IFN $\gamma-$ IL17+) CD4+ T cells generated after burn injury may be a novel cellular mechanism for postburn immunosuppression. J Trauma 2011;70:681-690.

3. Garner JS, Jarvis WR, Emori TG, Horan TC, Hughes JM. CDC definitions for nosocomial infections, 1988. Am J Infect Control 1988;16:128-140.

4. Centers for Disease Control and Prevention. National Healthcare Safety Network: tracking infections in acute care hospitals/facilities. 
http://www.cdc.gov/nhsn/acute-care-hospital/index.html. Accessed January 20, 2014.

5. Anderson DJ, Kirkland KB, Kaye KS, et al. Underresourced hospital infection control and prevention programs: penny wise, pound foolish? Infect Control Hosp Epidemiol 2007;28:767-773.

6. Weber DJ, Brown VM, Sickbert-Bennett EE, Rutala WA. Sustained and prolonged reduction in central line-associated bloodstream infections as a result of multiple interventions. Infect Control Hosp Epidemiol 2010;31:875-877.

7. Marschall J, Mermel LA, Classen D, et al. Strategies to prevent central line-associated bloodstream infections in acute care hospitals. Infect Control Hosp Epidemiol 2008;29(suppl 1):S22-S30.

8. King B, Schulman CI, Pepe A, Pappas P, Varas R, Namias N. Timing of central venous catheter exchange and frequency of bacteremia in burn patients. J Burn Care Res 2007;28:859-860.

9. Sood G, Heath D, Adams K, et al. Survey of central line-associated bloodstream infection prevention practices across American Burn Association-certified adult burn units. Infect Control Hosp Epidemiol 2013;34:439-440.

10. Harris AD, Pineles L, Belton B et al. Universal glove and gown use and acquisition of antibiotic-resistant bacteria in the ICU: a randomized trial. JAMA 2013;310:1571-1580.

\section{Microbial Contamination on Used Surgical Instruments}

Surgical instruments are considered critical items because they enter sterile body tissues or the vascular system, and if contaminated with any microorganism, including bacterial spores, this could result in an infection. Critical items are generally sterilized by steam sterilization if heat resistant. If heat sensitive, the object may be sterilized with ethylene oxide, hydrogen peroxide gas plasma, or vaporized hydrogen peroxide. However, these technologies have a lower margin of safety than steam sterilization. ${ }^{1}$ Because the level of microbial contamination of the object to be sterilized plays a critical role in determining the efficacy of the sterilization process, we evaluated the microbial load on used surgical instruments before cleaning and sterilization.

This study was conducted at the University of North Carolina Health Care, an 810-bed medical center. A variety of stainless steel surgical instruments were chosen, including Mayo straight scissors, forceps (eg, curved tip, large, and Debakey), rake, scissors (eg, curved), small-prong fork, smallpronged clamps, hemostats, knife handles, retractor, and needle holders. These instruments were all used in our operating rooms. After use in surgical procedures, the instruments were transported in peel packs to the hospital epidemiology laboratory and aseptically fully immersed in trypticase soy broth (Remel). The broth and instruments were agitated on a shaker at $150 \mathrm{rpm}$. After 30 minutes of agitation, two $500-\mu \mathrm{L}$ samples were removed and plated onto sheep blood agar (SBA; Remel). The remaining broth was filtered through a disposable $0.45-\mu \mathrm{m}$ cellulosic membrane filter unit (MSI Savur). Any colonies in the $500-\mu \mathrm{L}$ samples were identified, and the total number on the device was calculated by multiplying by the volume of fluid. Any colonies on the SBA or filter were enumerated and identified using standard microbiological techniques.

Fifty surgical instruments were obtained from 12 operations. Less than 10 colony-forming units (CFUs) per device were recovered from $58 \%$ of the used instruments (Table 1). Eleven to $100 \mathrm{CFU}$ were recovered from $20 \%$ of the used instruments, and greater than $100 \mathrm{CFU}$ (median, $207 \mathrm{CFU}$ ) were recovered from $14 \%$ of the instruments. In 4 cases ( $8 \%$ ), greater than 1,000 CFU were recovered from the instruments (ie, $2.4 \times 10^{3}$ Bacillus cereus on forceps; $4.4 \times 10^{3}$ B. cereus on a rake; $1.94 \times 10^{4}$ on a pronged clamp (containing $5.4 \times 10^{3}$ coagulase-negative Staphylococcus, $6.6 \times 10^{3} \alpha$ Streptococcus, $5.8 \times 10^{3}$ Streptococcus pneumoniae, and $1.6 \times 10^{3}$ Micrococcus species); and $4.98 \times 10^{4}$ on a hemostat (containing $5.4 \times 10^{3} \mathrm{~S}$. pneumoniae, $4.4 \times 10^{4} \alpha$-Streptococcus). The most common contaminating organisms were coagulase-negative Staphylococcus species, $\alpha$-Streptococcus, diphtheroids, Micrococcus species, B. cereus, Escherichia coli, and Bacillus species (Table 1).

The data revealed that the microbial load on used surgical instruments before cleaning was generally low; $58 \%$ had 10 CFU or less, and $78 \%$ had 100 CFU or less. In a study of contamination levels on used surgical instruments before

TABLE 1. Microbes Contaminating Used Surgical Instruments and Microbial Load When Submitted to Central Sterilization Services

\begin{tabular}{lc}
\hline Variable & $\begin{array}{c}\text { No. (\%) of } \\
\text { instruments } \\
(n=50)\end{array}$ \\
\hline Colony count, CFU & \\
$0-10$ & $29(58)$ \\
$11-100$ & $10(20)$ \\
$101-1,000$ & $7(14)$ \\
$>1,000$ & $4(8)$ \\
Microbe & \\
Coagulase-negative Staphylococcus species & $24(48)$ \\
$\alpha-S t r e p t o c o c c u s$ species & $13(26)$ \\
Diphtheroids & $10(20)$ \\
Micrococcus species & $10(20)$ \\
Bacillus cereus & $9(18)$ \\
Bacillus species (not cereus) & $5(10)$ \\
Escherichia coli & $5(10)$ \\
Enterococcus (vancomycin susceptible) & $2(4)$ \\
Pseudomonas aeruginosa & $2(4)$ \\
Streptococcus pneumoniae & $2(4)$ \\
Enterococcus species (vancomycin resistant) & $1(2)$ \\
Pantoea species & $1(2)$ \\
Serratia marcescens & $1(2)$ \\
Staphylococcus aureus (oxacillin susceptible) & $1(2)$ \\
\hline
\end{tabular}

NOTE. Instruments were used in a total of 12 operations. Of the 50 instruments cultured, 9 (18\%) showed no growth. CFU, colony-forming unit. 